\title{
Identification of Leishmania Species for Cutaneous leishmaniasis in Gonabad, Bardaskan and Kashmar, Central Khorasan, 2015
}

\author{
Abdolrahim Rezai, ${ }^{1}$ Elham Moghaddas, ${ }^{2}$ Mohammad Reza Bagherpor, ${ }^{2}$ Ali Naseri, ${ }^{2}$ and Seyed Aliakbar \\ Shamsian, $2,3, *$ \\ ${ }^{1}$ Department of Immunology, Faculty of Medicine, Mashhad University of Medical Sciences, Mashhad, IR Iran \\ ${ }^{2}$ Departments of Mycology and Parasitology, School of Medicine, Mashhad University of Medical Sciences, Mashhad, IR Iran \\ ${ }^{3}$ Iranian Academic Center for Education, Culture, and Research (ACECR), Mashhad, IR Iran \\ "Corresponding author: Seyed Aliakbar Shamsian, Department of Parasitology, School of Medicine, Mashhad University of Medical Sciences, Mashhad, IR Iran. Tel/Fax: \\ +98-5138002401-3, E-mail: ShamsianAA@mums.ac.ir
}

Received 2016 December 03; Revised 2016 February 26; Accepted 2017 March 08.

\begin{abstract}
Background: About $90 \%$ of cutaneous leishmaniasis (CL) cases are reported from 7 countries including Iran. In this study, the cutaneous leishmaniasis species causing CL in Gonabad, Bardaskan, Kashmar cities in central Khorasan (Iran) were identified by kDNA-PCR.

Methods: During the study, 93 suspected patients with CL, who were referred to the dermatology research center in these cities, were evaluated based on age, clinical forms, and place of residence. Direct microscopy was employed for parasitological diagnosis and PCR from skin ulcers performed using specific kDNA primers. Data were analyzed using the SPSS software.

Results: Among 93 individuals with skin ulcers suspected to CL, the results of 81 direct smears were positive. PCR bands were observed in 84 examined samples, of which 68 (81\%) samples were identified for Leishmania tropica and 16 (19\%) samples for L. major. 12 patients were from Bardaskan with 10 cases of L. tropica, 23 patients from Gonabad with 20 cases of L.tropica, and 49 patients from Kashmar with 38 cases of L. tropica. Most of the lesions were located on hands (37\%), the most clinical feature was papule (75\%), and most of patients were 21 - 30 years old.

Conclusions: Previous epidemiologic studies have indicated that anthroponotic cutaneous leishmaniasis is the only dominant CL in the center and south of Khorasan. However, this study introduced new foci of zoonotic cutaneous leishmaniasis in these areas. Kashmar city was introduced as a new focus of zoonotic cutaneous leishmaniasis.
\end{abstract}

Keywords: Cutaneous leishmaniasis, L. tropica, L. major, PCR, Iran

\section{Introduction}

Two species of Leishmania are responsible for cutaneous Leishmaniasis infections in Iran. Leishmania major and $L$. tropica are causative agents of zoonotic cutaneous leishmaniasis (ZCL) and anthroponotic cutaneous leishmaniasis (ACL), respectively. Both forms of this disease exist in Iran. Zoonotic cutaneous leishmaniasis is an endemic disease in 4 major regions of Iran: central parts (Isfahan province), north Iran (Semnan province), southwest Iran (Khozestan and Ilam provinces), and south Iran (Fars, Bushehr and Hormozgan provinces) (1,2). Anthroponotic cutaneous leishmaniasis is endemic in Tehran (capital city of Iran), Shiraz, Mashhad, and Kerman (3). It was reported that the 45 isolates of Leishmania from different geographical areas of Iran were suspected of cutaneous leishmaniasis (4). Every year it was reported that at least 20000 cases of cutaneous leishmaniasis are in Iran (5).

For many years, Khorasan province has a lot of foci of ACL and ZCL $(1,6)$. Leishmania tropica in most com- mon species that has been reported from Mashhad $(1,3)$, Khaf (5), Torbat-e Heydarieh (7), Kharve (8), Taybad (9), and Torghabeh - Shandiz (10). Sarakhs (11), Dargaz (unpublished data), Turkemen sahara (12), and Sabzevar (2) were introduced as L. major focuses.

\section{Objectives}

The aim of this study was to identify the presence of different species of Leishmania in most endemic areas of CL in Khorasan province including Gonabad, Bardaskan, Kashmar. There is no previous study regarding the leishmaniasis epidemiology in these cities.

\section{Methods}

\subsection{Study Area}

Kashmar is located near the river Sish Taraz in the western part of the province, 217 kilometers away from Mashhad; the capital of Khorasan province, with a population of 
81,527 inhabitants. Gonabad, located at 287 kilometers $\mathrm{km}$ distinct at the south of Mashed has a population of 36367 in habitats. The distance from Bardaskan to Mashhad is 288 kilometers at the margin of the north part of the salt desert (https://en.wikipedia.org/wiki/Kashmar). These cities have warm and dry climates with several seasonal rivers but no permanent river, as shown in Figure 1.

\subsection{Sampling}

In this cross-sectional study, people who had at least 1 skin ulcer suspected to CL were selected and introduced to clinical laboratories of the health center of Gonabad, Bardaskan, and Kashmar cities for direct examination. Prior to collecting, demographic and clinical information was obtained by a questionnaire. An informed consent form was obtained from each participant.

\subsection{Parasitological Examination}

To prepare direct Giemsa stained smears, samples were obtained by scraping from the center and edge of each skin lesion using a sterile scalpel (for each patient 2 smears). After air-drying, the slides were fixed in methanol, and stained with Giemsa (catalogue number: 1092040500). An expert microscopist examined each smear 2 times. Some scraping wound was retained in separately labeled containers in 70\% ethanol for molecular technique.

\subsection{Reference Strains}

Strains MRHO/IR/75/ER of L. major and the strain MHOM/IR/01/yaza of L. tropica were used in this study.

\subsection{DNA Extraction}

DNA extraction from tissue lesions was processed using the Ge Net Bio Kit (Korea), according to the manufacturer's protocol. The quantification and quality control of the DNA extraction procedures were performed using a nano spectrophotometer (NanoDrop 1000, Thermo Fisher Scientific). To avoid sample contamination, reactions were performed in appropriate places, following the good practice of laboratories.

\subsection{PCR for Leishmania kDNA}

A conventional PCR was used for the detection of Leishmania species and specific primers for kDNA were derived as forward (5'-TCGCAGAACGCCCCTACC -3') and reverse (5'AGGGGTTGGTGTAAAATAGG-3'), which was previously described by Motazedian et al. (13). Kinetoplast DNA was chosen as the molecular target, a $615 \mathrm{bp}$ fragment for L. major and $744 \mathrm{bp}$ fragment for L. tropica was amplified. The amplification conditions were $95^{\circ} \mathrm{C}$ for 5 minutes, followed by 40 cycles of denaturation at $94^{\circ} \mathrm{C}$ for 30 seconds, annealing at $60^{\circ} \mathrm{C}$ for 45 seconds, and extension at $72^{\circ} \mathrm{C}$ for 1 minute, with a final extension step at $72^{\circ} \mathrm{C}$ for 5 minutes. Two standard samples of parasites (L. tropica and L. major) and a negative control sample were used to monitor the reaction. Negative control contained primes, dNTP, $\mathrm{MgCl}_{2}$, Taq DNA polymerase, and distilled water. PCR products, by $100 \mathrm{bp}$ DNA Ladder(Fermentas), were visualized by EthBr staining following $2 \%$ agarose gel electrophoresis. The results were analyzed by SPSS 16 .

\subsection{Ethical Approval}

The experimental design for this study was approved by the ethics committee of Mashhad University of Medical sciences (IR.MUMS.REC.1392.805)

\section{Results}

\subsection{Sampling and Parasitological Examination}

Of the 93 samples obtained from direct smears of suspected patients, 81 were positive in parasitological examination while 84 (68 cases (81\%) L. tropica and 16 cases (19\%) L. major) were positive in PCR directed to kDNA as shown in Table 1. Among the rest of the patients that were negative for leishmaniasis ( 9 cases), 2 of them had the $M y$ cobacterium marinum infection on hands by culture on Lowenstein-Jensen medium. They are working in ornamental fish market. Among 84 individuals who had cutaneous leishmaniasis, 48 (57\%) were male and 36 (43\%) were female, where 37 (44\%) came from rural areas and 47 (56\%) were from urban regions. Most of the lesions were located on the hands (37\%), face (29\%), and feet (25\%). The most clinical features of skin lesions were papule $(75 \%)(\mathrm{P}=0.001)$ and ulcerated plaque (19\%). Most of the people with CL were 21 - 30 years, as shown in Figure 2.

Table 1. Results of the Diagnostic Methods for Cutaneous Leishmaniasis in Patients with Suspected Lesion(s) in Gonabad, Kashmar and Bardaskan During 2015

\begin{tabular}{lccc}
\hline Method & Positive & Negative & Total \\
\hline Giemsa stain & $81(87)$ & $12(13)$ & $93(100)$ \\
kDNA-PCR & $84(90)$ & $9(10)$ & $93(100)$ \\
\hline
\end{tabular}

${ }^{\mathrm{a}}$ Values are expressed as No. (\%).

\subsection{Molecular Diagnosis}

PCR method was performed for diagnosis and characterization of Leishmania species on extracted DNA obtained from suspected lesions. The results showed that 68 isolates, 68 patients ( $81 \%$ ) were L. tropica and 16 patients (19\%) were L. major. 12 patients were from Bardaskan (10 cases 

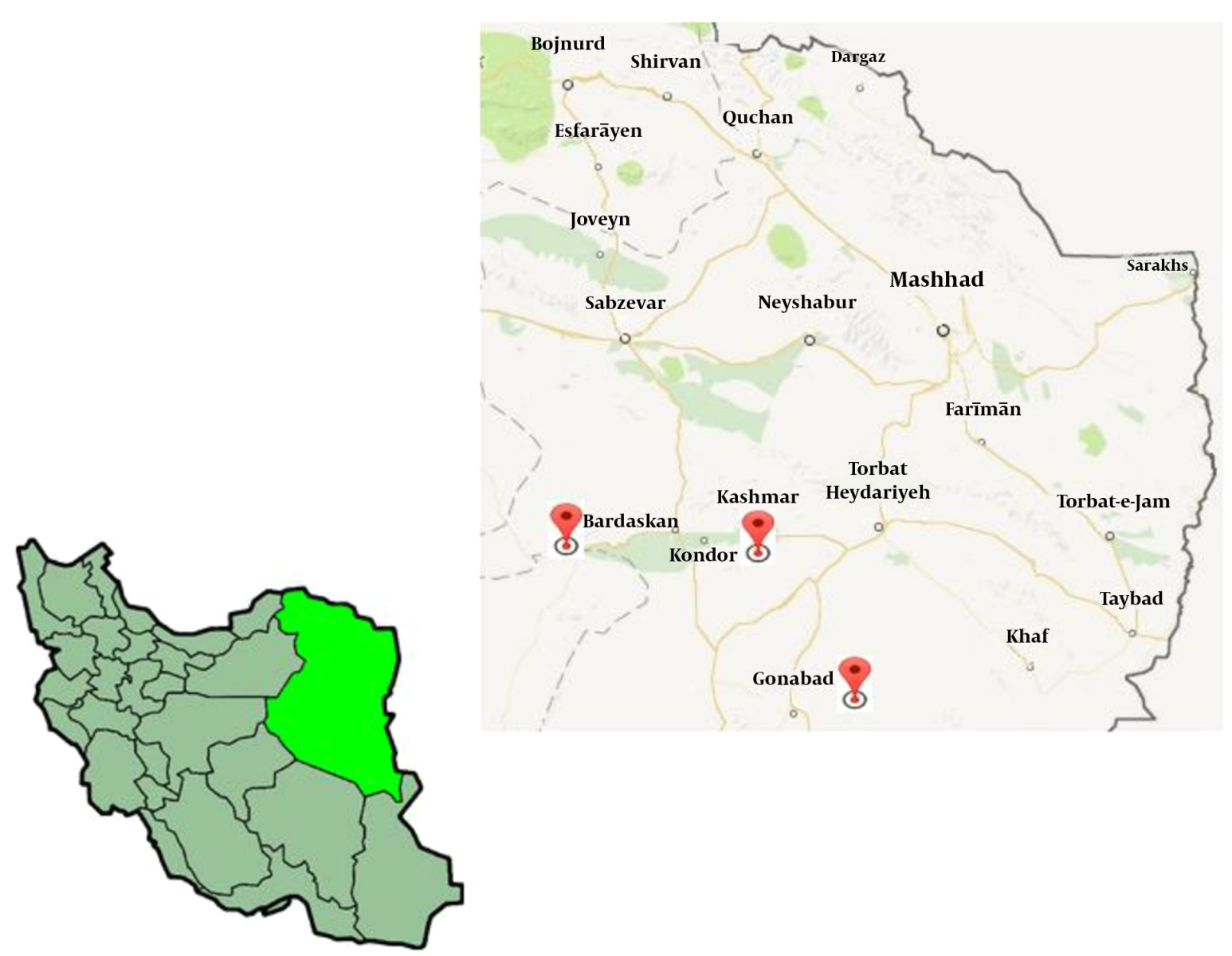

Figure 1. Geographical Map of Khasmar (K), Gonabad (G), and Bardaskan (B) Cities in Central Razavi, Iran.

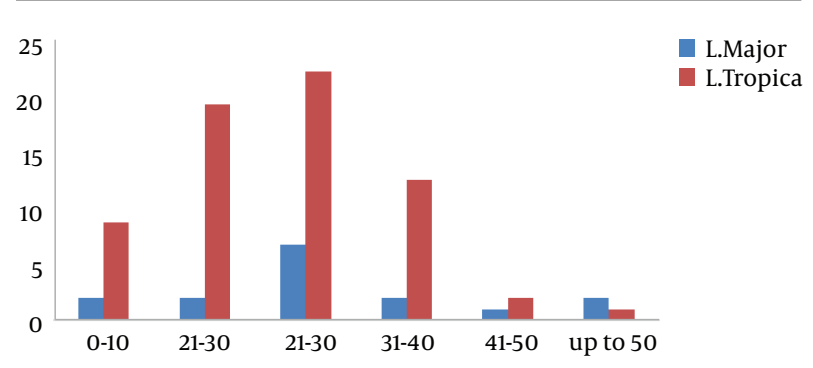

$\overline{\text { Figure 2. Frequency of Cutaneous Leishmaniasis in Different Age Groups of Patients }}$ in Gonabad, Kashmar and Bardaskan, During 2015

had L.tropica), 23 patients from Gonabad (20 cases had L.tropica), and 49 patients from Kashmar (38 cases had $L$. tropica). Kashmar was a focus of ZCL with $22.5 \%$ L. major (Table 2).
Table 2. Results kDNA-PCR for Cutaneous Leishmaniasis in Gonabad, Kashmar and Bardaskan During 2015

\begin{tabular}{lccc}
\hline Cities & Number of Patients & L.tropica Agent & L. major Agent \\
\hline Bardaskan & 12 & 10 & 2 \\
Gonabad & 23 & 20 & 3 \\
Kashmar & 49 & 38 & 11 \\
Total & 84 & 68 & 16 \\
\hline
\end{tabular}

\section{Discussion}

Characterization of Leishmania species and insect vectors is very helpful, due to the fact that different species have distinct treatment regimens and different prevention ways $(6,14)$. Intralesional injection of Glucantime was done more frequency for L. tropica (because of more drug resistance) rather than L. major. Furthermore, antibiotic 
therapy was proposed for treatment of superimposed bacterial wound in L. major lesions. It was reported that 4,900 CL cases were only from Mashhad (capital city of Khorasan province) and between 2000- 2002 with the most form of ACL (15). In another study from 21 positive samples, only 2 (9.5\%) isolates were L. major in Mashhad (1) and from the 136 smears by the PCR method all of the positive samples were L. tropica (10). However, the present study showed a prevalence of $19 \%$ for L. major in Gonabad, Bardaskan, and Kashmar cities in southeast Khorasan.

Some surrounding cities of Mashhad have been reported as ZCL focus such as Dargaz, Sabzevar, Sarakhs, and Turkemen sahara $(5,12,16)$. There was no information and exact data regarding etiological agents of leishmaniasis in above-mentioned cities. Already most patients who engaged with CL in these areas were ACL. Perhaps, Gonabad, Bardaskan, and Kashmar are small cities, they have close contacts between animal (Reservoir hosts of L. major), human, and Phlebotomus spp (vector host) rather than Mashhad city (the capital of Khorasan province). Therefore this study introduced a new foci of ZCL in the south and west of Khorasan. It was interesting to study the molecular diagnosis of Leishmania spp in rodents of this area. Almost all the cases of cutaneous leishmaniasis (90\%) occur in only 7 countries such as Iran, Afghanistan, and Saudi Arabia (17).

Khorasan province is the neighbor of Afghanistan, and every year, many Afghan refugees come to these cities for work. Apparently, such challenges increase the spread and focuses of the disease. In our study, most of the patients were male (57\%), similarly to other studies done in Isfahan, Shiraz, Damghan and Qom province in Iran $(18,19)$. The south and west of Khorasan has dry and warm weather, temperature above $40^{\circ} \mathrm{C}$ in the summer and near the great desert. Due to these conditions men who work in deserts and wastelands and wear fewer parts of clothing probably have more contact with sand flies during the evening rather than women. In the present study, most of the lesions were located on hands (37\%); other studies in various parts of Iran have also suggested that the most of lesions of ACL occur in hands $(20,21)$.

kDNA- PCR sensitivities was described between $75 \%$ 98\% for detection of Leishmania species in many studies (22-25). Even kDNA was detected in the urine of patients with cutaneous leishmaniasis (26) and with visceral leishmaniasis (27). Recently, for the first time, real-time PCR was used for diagnosis and identification of Leishmania spp with a sensitivity of 98\% (96/102) on Giemsa-stained slides that were stored for more than 3 years (28).

In this study, the results of kDNA-PCR approved that both diseases (ACL and ZCL) exist in the cities of Gonabad, Kashmar, and Bardeskan. Moreover, it seems that Mashhad is the important foci of ACL, while ZCL is more prevalent in the cities around Mashhad. Surrounding cities of Mashhad could be an important reservoir of ZCL for the Khorasan Province as a pilgrimage center for Muslims.

\subsection{Conclusion}

These data indicate that both L. tropica and L. major are the causative agents of cutaneous leishmaniasis in the Khorasan province. L. tropica is the dominant Leishmania species in Mashhad but in smaller cities of the Khorasan province ZCL focuses exists. Travelers in endemic areas should be advised that the only preventive measures are those of protection against sandflies bite. Leishmaniasis should be considered in travelers with compatible clinical findings and a history of travel to an endemic area, even months or years ago. Information regarding leishmaniasis and appropriate protective measures should be offered to adventure travelers, military personnel, and immigrants likely to be exposed to sandflies in endemic areas.

\section{Acknowledgments}

We thank colleagues' laboratory and the staff of Gonabad, Kashmar and Bardaskan health centers. We are thankful to Mashhad University of Medical sciences for financially supporting this study that is obtained from MS thesis (grant number: 921466).

\section{Footnotes}

Authors' Contribution: Study concept, design, and critical revision of the manuscript: Aliakbar Shamsian and Ali Naseri; analysis and interpretation of data: Mohammad Reza Bagherpor; drafting of the manuscript: Elham Moghaddas; statistical analysis: Abdolrahim Rezaee.

Funding/Support: Financial support for this study was provided by Mashhad University of Medical sciences.

Conflict of Interest: The authors report no conflicts of interest. The authors alone are responsible for the content and writing of the paper.

\section{References}

1. Reza MM, Masoud M, Jalil TA, Taghae SM, Javad YP, Fariba B, et al. Molecular identification of Leishmania species causing cutaneous leishmaniasis in Mashhad, Iran. Jundishapur J Microbiol. 2010;3(4):195-200.

2. Mohajery M, Shamsian SA, Rezaee A, Hasan PK, Shakeri MT, Farnoosh $G$, et al. Evaluation of mulecular epidemiology of cutaneous leishmaniasis in Sabzevar. Med J Mashhad Univ Med Sci. 2010;53(3):138-44.

3. Tashakori M, Ajdary S, Kariminia A, Mahboudi F, Alimohammadian $\mathrm{MH}$. Characterization of Leishmania species and L. major strains in different endemic areas of cutaneous leishmaniasis in Iran. Iran Biomed J. 2003;7(2):43-50. 
4. Hatam GR, Riyad M, Bichichi M, Hejazi SH, Guessous-Idrissi N, Ardehali S. Isoenzyme characterization of Iranian Leishmania isolates from cutaneous leishmaniasis. Iran J Sci Technol. 2005;29(1):65-70.

5. Abdolmajid F, Ghodratollah SS, Hushang R, Mojtaba MB, Ali MM, Abdolghayoum M. Identification of Leishmania species by kinetoplast DNA-polymerase chain reaction for the first time in Khaf district, Khorasan-e-Razavi province, Iran. Trop Parasitol. 2015;5(1):50-4. doi: 10.4103/2229-5070.145587. [PubMed: 25709953].

6. Valizadeh M, Dalimi ASLH, Fata AAM, Jafari MR, Khamesipour A, Mohebali M. A study on leishmania species causing cutaneous leishmaniasis inmashhad using specific monoclonal antibodies. Modares J Med Sci. 2005;7(2):107-13.

7. Naseri A, Fata A, Rezai A, Hedayatimoghadam M, Berengi F, Akbarzadeh $\mathrm{O}$, et al. Molecular identification of Leishmania species in Torbat-e Heydarieh, Khorasan Razavi province, Iran. Health Sci. 2016;5(6):87-92.

8. Saadabadi F, Mohajery M, Poostchi E, Shamsian SA. Identification of Leishmania species causing cutaneous leishmaniasis using Random Amplified Polymorphic DNA (RAPD-PCR) in Kharve, Iran. Rep Biochem Mol Biol. 2013;1(2):69-73. [PubMed: 26989711].

9. Ghodratollah SA, bdolmajid F, Ali MM, Mojtaba MBS, Hushang R, Abdolghayom M. Molecular identification of Leishmania species in Taybad district, Iran. Asian Pac J Trop Dis. 2014;4:S535-9.

10. Farash BRH, Mohajery M, Fata A, Shamsian SA, Rezaee A, Yazdanpanah MJ. Anthroponotic cutaneous leishmaniasis in torghabeh-shandiz, a region with rural texture (a molecular study). Jundishapur J Microbiol. 2013;6(10).

11. Shamsian S, Rezaei A, Akbarzadeh A, Hosseini Farash B. Molecular Identification of Leishmania tropica in an Endemic Border City for Zoonotic Cutaneous Leishmaniasis (ZCL) in Northeastern Iran. J Microb Exp. 2015;2(4):53-8.

12. Mirzaei A, Rouhani S, Taherkhani H, Farahmand M, Kazemi B, Hedayati $\mathrm{M}$, et al. Isolation and detection of Leishmania species among naturally infected Rhombomis opimus, a reservoir host of zoonotic cutaneous leishmaniasis in Turkemen Sahara, North East of Iran. Exp Parasitol. 2011;129(4):375-80. doi: 10.1016/j.exppara.2011.08.020. [PubMed: 21945269].

13. Motazedian H, Karamian M, Noyes HA, Ardehali S. DNA extraction and amplification of leishmania from archived, Giemsa-stained slides, for the diagnosis of cutaneous Leishmaniasis by PCR. Ann Trop Med Parasitol. 2002;96(1):31-4. doi: 10.1179/000349802125000484. [PubMed: 11989531].

14. Kazemi-Rad E, Mohebali M, Hajjaran H, Rezaei S, Mamishi S. Diagnosis and characterization of Leishmania species in Giemsa-stained slides by PCR-RFLP. Iran J Public Health. 2008;37(1):54-60.

15. Hajjaran H, Mohebali M, Razavi MR, Rezaei S, Kazemi B, Edrissian G, et al. Identification of Leishmania species isolated from human cutaneous leishmaniasis, using random amplified polymorphic DNA (RAPD-PCR). Iran J Public Health. 2004;33(4):8-15.
16. Mohaghegh M, Fata A, Salehi G, Berenji F, Bazzaz MM, Rafatpanah H, et al. Molecular identification of leishmania species using samples obtained from negative stained smears. Iran J Parasitol. 2013;8(2):337-41. [PubMed: 23914250].

17. Ali MM, Mohammad O. Intralesional injections of metronidazole versus meglumine antimoniate for the treatment of cutaneous leishmaniasis. Jundishapur J Microbiol. 2010;3(2):79-83.

18. Rafati N, Shapori-Moghadam A, Ghorbani R. Epidemiological survey of cutaneous leishmaniasis in Damghan (1999-2005). J Semnan Univ Med Sci. 2004;2(1):247-53.

19. Karami M, Doudi M, Setorki M. Assessing epidemiology of cutaneous leishmaniasis in Isfahan, Iran. J Vector Borne Dis. 2013;50(1):30-7. [PubMed: 23703437].

20. Hatam GR, Rezanezhad H, Motazedian MH, Sarkari B. In vitro infectivity of Leishmania major isolated from patients with different clinical forms of cutaneous leishmaniasis and its association with parasite zymodems. Iran J Parasitol. 2009;4(3):52-60.

21. Gramiccia M, Gradoni L. The current status of zoonotic leishmaniases and approaches to disease control. Int J Parasitol. 2005;35(11-12):116980. doi: 10.1016/j.ijpara.2005.07.001. [PubMed: 16162348].

22. Bensoussan E, Nasereddin A, Jonas F, Schnur LF, Jaffe CL. Comparison of PCR assays for diagnosis of cutaneous leishmaniasis. J Clin Microbiol. 2006;44(4):1435-9.

23. Oliveira DM, Lonardoni MV, Teodoro U, Silveira TG. Comparison of different primes for PCR-based diagnosis of cutaneous leishmaniasis. Braz J Infect Dis. 2011;15(3):204-10. [PubMed: 21670918].

24. El-Beshbishy HA, Al-Ali KH, El-Badry AA. Molecular characterization of cutaneous leishmaniasis in Al-Madinah Al-Munawarah province, western Saudi Arabia. Int J Infect Dis. 2013;17(5):e334-8. doi: 10.1016/j.ijid.2012.11.015. [PubMed: 23253909].

25. Nicodemo AC, Amato VS, Tuon FF, Souza RM, Okay TS, Braz LM. Usefulness of kDNA PCR in the diagnosis of visceral leishmaniasis reactivation in co-infected patients. Rev Inst Med Trop Sao Paulo. 2013;55(6):429-31. doi: 10.1590/S0036-46652013000600011. [PubMed: 24213198].

26. Veland N, Espinosa D, Valencia BM, Ramos AP, Calderon F, Arevalo $\mathrm{J}$, et al. Polymerase chain reaction detection of Leishmania kDNA from the urine of Peruvian patients with cutaneous and mucocutaneous leishmaniasis. Am J Trop Med Hyg. 2011;84(4):556-61. doi: 10.4269/ajtmh.2011.10-0556. [PubMed: 21460009].

27. Fisa R, Riera C, Lopez-Chejade P, Molina I, Gallego M, Falco V, et al. Leishmania infantum DNA detection in urine from patients with visceral leishmaniasis and after treatment control. Am J Trop Med Hyg. 2008;78(5):741-4. [PubMed: 18458307].

28. Khademvatan S, Neisi N, Maraghi S, Saki J. Diagnosis and identification of Leishmania spp. from Giemsa-stained slides, by real-time PCR and melting curve analysis in south-west of Iran. Ann Trop Med Parasitol. 2011;105(8):559-65. doi: 10.1179/2047773211Y.0000000014. [PubMed: 22325815]. 